\title{
- - Antropología Etnobotánica Etnomedicina
}




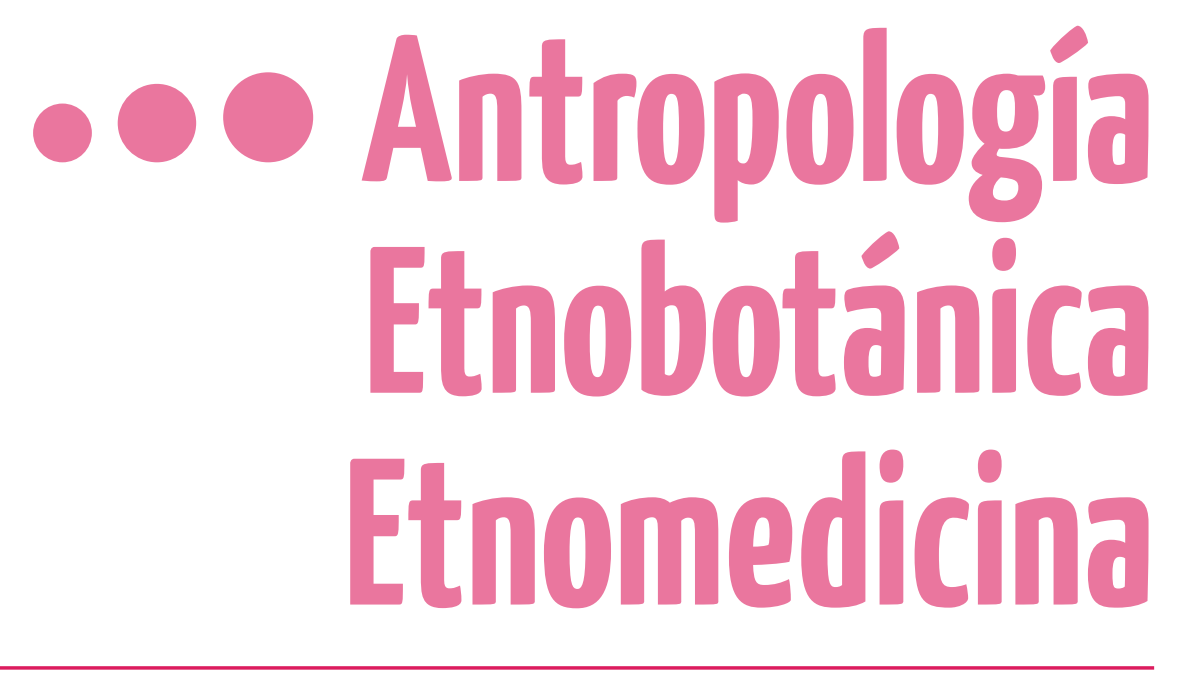

Presentaciones Orales 


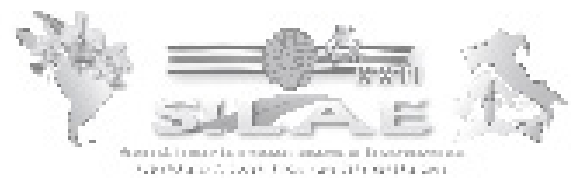

\title{
ETNOMEDICINA WAYUU: UNA ALTERNATIVA EN LA CURA Y PREVENCIÓN DE ENFERMEDADES DE LAS COMUNIDADES INDÍGENAS DEL DEPARTAMENTO DE LA GUAJIRA, COLOMBIA
}

\author{
Jairo Rafael Rosado Vega \\ Universidad de Guajira, Colombia
}

\section{INTRODUCCIÓN}

El objetivo de la presente investigación fue evaluar e identificar las plantas medicinales, sus preparaciones y usos en la cura y prevención de enfermedades de la comunidad Wayuu y realizar una compilación sobre su Cosmovisión desde la visión de los diferentes autores con conocimientos sobre la Farmacopea Guajira, pretendiendo con ello, contribuir al rescate de los saberes tradicionales entre las comunidades indígenas y permitiendo su difusión en las nuevas generaciones.

\section{METODOLOGÍA}

Se realizaron encuestas a los Ouutsü y Alüjülii asentados en las zonas de muestreo de los municipios de Riohacha, Maicao y Uribia, sobre las formas de preparación y usos de las plantas medicinales y luego se identificaron botánicamente, procediendo de inmediato a la tabulación y comparación de los resultados en las diferentes zonas de muestreo. Después se optó por realizar una revisión bibliográfica exhaustiva de los PA contenidos en un gran número de ellas y determinar así su asociación con las acciones terapéuticas reportadas por las comunidades. Así mismo, se hizo una compilación de las diferentes publicaciones sobre la Cosmovisión Wayuu, sus creencias y saberes con respecto a las enfermedades y sus relaciones con las plantas.

\section{RESULTADOS}

Los resultados indican que existen 155 plantas asociadas en la cura de 175 enfermedades que aquejan a los Wayuu, alcanzando mayor frecuencia el dolor de estómago (20.0\%), infección dérmica (18.7\%), fiebre (16.1\%), diarrea y gripa (15.5\%). Las plantas que alcanzaron los porcentajes más altos en la cura y prevención de dichas enfermedades fueron: Croton malambo (13.7\%), Aloe barbadensis (11.4\%), Castela erecta (10.3\%), Prosopis juliflora (9.7\%), Jatropha urens (9.1\%), Libidibia coriaria y Arrabidea chica (8.6\%); utilizándose en mayor proporción la planta entera $(25.6 \%)$, hojas $(24.3 \%)$, raíces $(13.3 \%)$ y tallos $(9.1 \%)$ mediante la cocción $(26.8 \%)$, infusión (13.3\%), trituración (11.7\%) y mezcla con otras plantas (11.0\%). Las formas más comunes de utilizarlas fueron: bebida (39.6\%), aplicación directa de la planta (20.8\%), baños (15.5\%) y masajes (11.3\%). Los grupos de principios activos que están presentes en mayor proporción en las plantas medicinales fueron: glucósidos (57.1\%), terpenos (51.0\%), alcaloides (34.7\%), flavonoides (30.6\%) y taninos (27.9\%).

\section{CONCLUSIONES}

Existe una gran diversidad florística asociada a la etnobotánica Wayuu y se hace necesario fomentar este acervo de conocimientos entre las comunidades indígenas mediante la construcción de Jardines botánicos y la difusión masiva de materiales didácticos en lengua Wayuunaiki dirigidos a estas comunidades y al público en general.

\section{PALABRAS CLAVES}

Plantas medicinales, Cosmovisión Wayuu, etnobotánica, Ouutsü, Alüjülii. 


\section{GRANOS ANDINOS ALIMENTO MILENARIO}

\section{Katia Peralta Hinojosa' y Artemio Chang Canales²}

1. Instituto Peruano de Investigación Fitoterápica Andina (Ipifa); kperaltah@terra.com.pe

2. Facultad de Farmacia Universidad San Luis Gonzaga Ica; achang@yahoo.es

\section{INTRODUCCIÓN}

El Perú es uno de los 10 países con mayor biodiversidad del planeta, posee 84 zonas de vida y 28 tipos de clima, que posibilitan la existencia de un impresionante variedad de especies de flora, muchas de ellas aún desconocidas por la ciencia.

La Quinua (Chenopodium quinoa) juntamente con la Kañiwua (Chenopodium pallidicaule) y la Kiwicha (Amaranthus caudatus) son granos andinos sumamente nutritivos,crecen en la zona del altiplano, junto al lago Titicaca. Es también un tema histórico, revisando los restos arqueológicos se sabe que la quinua tiene mas de 4 mil años de antigüedad y su consumo se expandió a lo largo del Imperio Inca a través del Capac Ñan, de modo que además del Perú llegó hasta Ecuador,Colombia,Chile y el norte de Argentina, a lo largo de miles de años los antiguos peruanos, acumularon importantes conocimientos acerca de los usos y propiedades de las especies nativas. En la actualidad, el mercado mundial se concentra en la búsqueda de alimentos orgánicos,saludables y nutritivos.

\section{METODOLOGÍA}

Los datos fueron obtenidos por medio de entrevistas con las personas lugareñas (zona andina), levantamiento de datos,colecta de los granos para su posterior degustación en los comedores populares. Las muestras fueron remitidas al laboratorio para su identificación botánica,y análisis fitoquímico de rutina.

\section{RESULTADOS Y DISCUSIÓN}

Los granos andinos se caracterizan por contener proteínas de alto poder biológico (aminoácidos esenciales) sus propiedades nutricionales son la principal razón de su consumo ancestral y la actual demanda internacional.

\section{Composición}

\begin{tabular}{|c|c|c|c|}
\hline & Quinua & Kañiwa & Kiwicha \\
\hline Proteinas & 1.7 & 14.0 & 12.9 \\
\hline Grasa & 6.3 & 4.3 & 7.2 \\
\hline Carbohidrato & 68.0 & 64.0 & 65.1 \\
\hline Fibra & 5.2 & 9.8 & 6.7 \\
\hline Ceniza & 2.8 & 5.4 & 2.5 \\
\hline Humedad \% & 11.2 & 12.2 & 12.3 \\
\hline
\end{tabular}

Fuente: Collazos et al. 1995

\section{CONCLUSIONES}

La ONU ha declarado el 2013 como el año internacional de la Quinua,esto implica el rescate de estos granos que estaban durmiendo entre las comunidades andinas, que son las que han conservado todo el material genético de estos cultivos. La Quinua tiene siete aminoácidos básicos para el buén desarrollo intelectual del niño menor de cinco años, por lo tanto cambiemos las galletas de soda de la lonchera por barritas de grano andino.

\section{BIBLIOGRAFIA}

Repo-Carrasco,R,1992 Cultivos Andinos y la alimentación infantil. Lima Perú

Tapia,M. 1993.Granos Andinos, el banco de oro. Concytec. Lima Perú

Brack Egg 1997,Diccionario de Plantas Medicinales Andino Amazónicas. Lima Perú 


\title{
INDUCCIÓN DE EMBRIOGÉNESIS SOMÁTICA DE Phytolacca tetramera, ESPECIE MEDICINAL DE ARGENTINA
}

\author{
Basiglio Cordal, M.A; Adema, M; Briones, V; Villarreal, B; Abedini, W. y Sharry, S \\ Centro Experimental de Propagación Vegetativa (C.E.Pro.Ve). Facultad de Ciencias Agrarias y Forestales. Universidad Nacional \\ de La Plata. CICPBA- CONICET. Diagonal 113 Nº 469 (1900) La Plata, Buenos Aires, Argentina; maribasiglio@hotmail.com
}

\section{INTRODUCCIÓN}

Phytolacca tetramera es una especie endémica de la Prov. de Buenos Aires (Argentina), en peligro de extinción. Posee principios activos funguicidas. El extracto metanólico de sus bayas, posee actividad antifúngica contra hongos patógenos oportunistas. El fraccionamiento del extracto butanólico, permitió el aislamiento de 3 saponinas triterpenoides monodesmosídicas: fitolacósidos B [3-O-B-d-xylopiranosyl-phytolaccagenin], E [3-O-Bd-glucopyranosyl- $(1 \rightarrow 4)$ - B-d-xylopiranosyl-phytolaccagenin] y $F$ [3-O- $\alpha$-l-rhamnopyranosyl- $(1 \rightarrow 2)$ - $\beta-d-$ glucopyranosyl- $(1 \rightarrow 2)$ - ácido $B$-d-xylopyranosyl-phytolaccagenic]. El primero de estos compuestos, produjo malformaciones de las hifas y un engrosamiento de la membrana fúngica en Saccharomyces cerevisiae (Escalante et al; 2002). La técnica de embriogénesis somática se basa en la formación de un embrión a partir de una célula somática, es decir, sin la necesidad de la fusión de gametos (Tisserat et al, 1979), lo que facilita la producción masiva de plantas in vitro. La obtención de embriones somáticos permite automatizar el proceso productivo, en cortos periodos de tiempo y en un espacio reducido. La técnica es un paso necesario para generar semillas sintéticas. En Phytolacca tetrámera, la embriogénesis somática, o el cultivo de células en medio liquido con posterior regeneración de plantas, seria un proceso de vital importancia, ya que, dicha especie se destaca por estar en peligro crítico de amenaza.

\section{METODOLOGÍA}

Se utilizaron como explantos secciones de hojas jóvenes de plantas de Phytolacca tetrámera obtenidas in vitro. Se colocaron en el medio de cultivo MS (Murashige-Skoog, 1962) suplementado con 2,4 D (2,4-diclorofenoxiacetico) en diferentes concentraciones (0ppm $=$ Testigo; $0,5 \mathrm{ppm} ; 1 \mathrm{ppm} ; 1,5 \mathrm{ppm})$ con 4 repeticiones por tratamiento. Los explantos fueron incubados en oscuridad a $25+/-2{ }^{\circ} \mathrm{C}$ grados de temperatura. A los 20 días, los callos formados en el borde de corte de la hoja fueron subcultivados a medio MS líquido en agitación y los callos formados en la nervadura principal fueron subcultivados a un medio MS sólido. Los pro-embriones y los embriones somáticos en estadios tempranos fueron subcultivados a un medio MS semisólido sin reguladores de crecimiento.

\section{RESULTADOS}

A los 6 días de iniciado el ensayo, los explantos colocados en MS con el agregado de 0,5; 1 y 1,5 ppm de 2,4D comenzaron a desdiferenciarse. A los 20 días de cultivo, los explantos formaron callos en la superficie a nivel de la nervadura principal y en el borde o superficie de corte. Los callos formados a nivel de la nervadura eran friables y de color blanco. Estas son características típicas de los calos embriogénicos. En los mismos se identificaron embriones en estadio globular. Los callos formados en los bordes del explante, eran disgregables, y se pudieron observar algunos embriones somáticos en diferentes estadios (globular y torpedo).

\section{CONCLUSIONES}

Los resultados indican que se ha podido inducir la formación de callos embriogénicos y embriones somáticos a partir de hojas de Phytolacca tetrámera. Esta técnica nos permitirá tener una gran cantidad de plantas para introducir en su hábitat natural y domesticar esta especie para establecer cultivos a campo con el objeto de obtener principios activos de interés fitomedicinal. 


\section{BIBLIOGRAFÍA}

Escalante, AM; Santecchia, CB; López, SN; Gattuso, MA; Gutierrez Ravelo, A; Delle Monache, F; Gonzalez Sierra, M; Zacchino, SA. 2002. Isolation of antifungal saponins from Phytolacca tetramera an Argentinean species in critic risk. Journal of Ethnopharmacology.

Murashige T \& Skoog F. 1962. A revised medium for rapid growth and bioassays with tobacco tissue cultures. Physiol. Plant. 15:473-97.

Tisserat, B, Esan E y Murashige T (1979) Somatic embryogenesis in angiosperms. Hort. Rev. 1: 1-78 


\title{
DETERMINACIÓN DE COMPONENTES CON POTENCIAL BIOACTIVO DE CUATRO PLANTAS COSTARRICENSES CON USO TRADICIONAL CONOCIDO.
}

\author{
Arnaéz, E. ${ }^{1}$; Navarro, M. ${ }^{2}$; Moreira, I. ${ }^{1}$; Quesada, S. ${ }^{2}$; Monagas, M. ${ }^{3}$; Murillo, R. ${ }^{2}$; Castro, V. ${ }^{4}$; \\ Zamora, W. ${ }^{4}$; Cordero, M. ${ }^{5}$; Loaiza, J.6; Alarcón, Y. ${ }^{7}$ \\ 1. M.Sc/Profesora, Instituto Tecnológico de Costa Rica, Cartago, Costa Rica; earnaez@itcr.ac.cr \\ 2. Ph.D./Profesor(a), Universidad de Costa Rica, San José, Costa Rica; mnavarro@codeti.org \\ 3. Ph.D./Profesora, CIAL, Consejo Superior de Investigaciones Científicas, Madrid, España \\ 4. Lic./Profesor, Universidad de Costa Rica, San José, Costa Rica \\ 5. Lab./Profesora, Universidad Estatal a Distancia, Campus Universitario de Alajuela, Costa Rica \\ 6. PhD/Profesor, Universidad Nacional, Centro Universitario de Sarapiquí, Heredia, Costa Rica \\ 7. M.Sc/Investigadora, Universidad Nacional, Heredia, Costa Rica
}

\section{INTRODUCCIÓN}

Estudios asociados al conocimiento sobre el uso de plantas en medicina tradicional han permitido la aplicación de un modelo de investigación multidisciplinaria que combina métodos científicos con transferencia tecnológica a comunidades rurales para contribuir a su desarrollo económico sostenible a través del aprovechamiento racional de plantas con potencial medicinal. Este modelo, que incluye la evaluación de las condiciones de producción natural y con manejo agroecológico de las especies, la determinación del nivel de producción de biomasa a dos y tres años, el desarrollo de estudios fitoquímicos y de bioactividad así como la capacitación interdisciplinaria productiva de comunidades meta en forma sostenible con el ambiente, fue aplicado en relación a cuatro plantas, Phyllanthus niruri (chancapiedra o riñoncillo), Senna reticulata (saragundí), Pettiveria alliaceae (ajillo) y Phyllanthus acuminatus (chilillo), habiéndose trabajado con comunidades de las regiones Huetar Atlántica y Huetar Norte de Costa Rica.

\section{METODOLOGÍA}

Las especies se georeferenciaron, separaron sus partes, se secaron y trataron para la elaboración de extractos con solventes de diferente polaridad, procediéndose a su fraccionamiento y análisis, mediante técnicas de UPLCDAD/TQ-ESI MS y de RMN. Asimismo se determinó la actividad antioxidante a través de dos metodologías, DPPH y ORAC, así como la citotoxicidad en líneas celulares cancerígenas con el método MTT.

Por otro lado, con el fin de proteger estas especies, se procedió a su multiplicación por medio de propagación sexual o vegetativa convencional, se inició el proceso de domesticación de las especies seleccionadas y su estudio agroecológico, se potenció la elaboración de productos, unido a la capacitación y acompañamiento a las comunidades.

\section{RESULTADOS Y DISCUSIÓN}

Los resultados de los análisis realizados en las cuatro especies, su interpretación y sistematización permitieron elucidar estructuras con potencial bioactivo, como lignanos, tetraterpenos, fitosteroles, polifenoles monoméricos glicosidados, agliconas y flavan-3-oles, incluyendo dímeros de procianidina y propelargonidina; así como determinar el contenido total de polifenoles (Folin-Ciocalteau) y de procianidinas. Así como correlacionar este contenido con los resultados de cromatografía y espectroscopia de masas; obteniéndose asimismo valores de IC50 de potencial antioxidante y anticancerígeno en líneas de cáncer gástrico (AGS) y de colon (SW620).

Asimismo, se realizaron la estimación de la biomasa de las especies, su propagación, talleres con la participación de grupos comunales de las dos regiones, entrenándoseles en buenas prácticas agrícolas y de recolección así como productivas acordes a los lineamientos de la Organización Mundial de la Salud, lográndose complementariamente establecer rutas de visitas agroecológicas, viveros y jardines de plantas medicinales, así como preparación de extractos de las plantas estudiadas y su aplicación en formulaciones mejoradas de jabón líquido, geles y cremas. 


\section{CONCLUSIÓN}

Esta investigación buscó a través del método científico, validar las propiedades fitoquímicas y actividad biológica potencial de Phyllantus niruri (chanca piedra), Senna reticulata (saragundí), Pettiveria alliaceae (ajillo) y Phyllantus acuminata (chilillo), mediante técnicas de laboratorio que permiten avanzar en el establecimiento de protocolos que aseguren el buen uso de las mismas; asimismo con el fin de que este conocimiento pueda ser usado como base para la elaboración mejorada de algunos fitofármacos tópicos. Al mismo tiempo, de forma sistematizada, se introdujeron buenas prácticas de cultivo y productivas específicas para las cuatro especies en los grupos meta en las regiones Huetar Norte y Huetar Atlántica, de forma a que puedan hacer uso sostenible de las mismas.

\section{BIBLIOGRAFÍA}

García G.M. et al. (2006) Subchronic and acute preclinic toxicity and some pharmacological effects of the water extract from leaves of Petiveria alliacea (Phytolaccaceae) Rev. Biol. Trop. 54 (4): 1323-1326. Dec. 15.

Germosén-Robineau, L (ed.). (2005). Farmacopea vegetal caribeña, TRAMIL. Segunda edición. Editorial Universitaria UNANLeón, Nicaragua.

Jiménez, A. (2002). Caracterización de 10 genotipos de saragundí (Senna reticulata) propagación vegetativa por estacas y desarrollo de productos en Pocora, Tesis para optar al grado de Licenciatura de la Universidad EARTH. Costa Rica.

Jiménez, M.; Alvarenga, S.; Alan, E. (2007). Establecimiento de protocolo de micropropagación para la planta medicinal Phyllanthus niruri (Euphorbiaceae). Tecnología en Marcha 20(2): 32-40.

Mdlolo C.M., Shandu J.S. and Oyedeji O.A. 2008. Phytochemical Constituents and Antimicrobial Studies of Two South African Phyllantus species. African. Journal of Biotechnology Vol. 7 (5), pp. 639-643, 4 March.

Pettit, G.R. et al. (1984). Antineoplasic Agents. 104. Isolation And Structure Of The Phyllantus acuminatus Vahl (Euphorbiaceae) Glycosides J. Org. Chem. Vol. 49, No. 22, 4258-4266.

Phyllanthus acuminatus. Tropicos.org.Missouri Botanical Garden. Consultado el 4 de diciembre de 2012. 


\title{
EVALUACIÓN DE LA BIOACTIVIDAD DEL FOLLAJE DE DOS ESPECIES AGROFORESTALES: Eucalyptus pellita Y Copaifera officinalis, PARA LA OBTENCIÓN DE PRINCIPIOS ACTIVOS CON APLICACIÓN EN LA INDUSTRIA
}

\author{
Ludy C. Pabón, Margarita Rendón, Alexandra Delgadillo, Patricia Hernández-Rodríguez \\ Departamento de Ciencias Básicas de La Universidad de La Salle. Bogotá, Colombia; lupabon@unisalle.edu.co; \\ phernandez@unisalle.edu.co \\ *Proyecto Financiado por la Vicerrectoria de Investigación y Transferencia (VRIT). Universidad de La Salle.
}

\section{INTRODUCCIÓN}

Las especies agroforestales además del uso maderable presentan aceites esenciales, extractos, harinas vitamínicas y principios activos de utilidad en la industria farmacéutica, alimenticia y agrícola. Para otorgar un valor agregado a estos recursos es necesario conocer la composición química y el potencial biológico; por tanto, este trabajo tuvo como objetivo caracterizar química y biológicamente los aceites esenciales y extractos etanólicos del follaje de Eucalyptus pellita y Copaifera officinalis.

\section{METODOLOGÍA}

Se extrajeron los aceites esenciales por arrastre con vapor, y extractos etanólicos por maceración en frío. Los metabolitos secundarios se determinaron por análisis fitoquímico y los lípidos, proteínas, fibra cruda y compuestos fenólicos se cuantificaron por saponificación, khjendal e hidrólisis ácido-base y espectrofometria respectivamente. La capacidad antioxidante se evaluó por el método bioautográfico empleando como revelador B-caroteno y para evaluar la actividad antibacterial se utilizaron cepas ATCC de Salmonella infantis, Escherichia coli y Staphylococcus aureus. El potencial herbicida se estableció mediante el porcentaje de inhibición (EC50) en semillas de lechuga (Lactuca sativa) y trébol (Trifolium repens).

\section{RESULTADOS}

Los resultados determinaron un rendimiento de extracción para los aceites de E. pellita y C. officinalis de 0.2 y $0.06 \%$, y para los extractos de 19 y $6.4 \%$; las dos especies presentaron esteroides flavonoides, taninos y saponinas como principales metabolitos secundarios. Se encontraron índices de saponificación entre 200 y $260 \mathrm{mg}$ de KOH/g de muestra y se observó un aumento en el contenido de lípidos durante la extracción con etanol. El porcentaje de fibra de E. pellita $(39,6 \%)$ fue el doble del hallado en C. officinalis $(19.5 \%)$ y el porcentaje de proteína fue del $10 \%$ para ambas especies antes de la extracción con etanol y luego de la extracción disminuye notablemente para C. oficinallis. Los extractos etanólicos presentaron el mayor contenido de compuestos fenólicos (150 y 170 mg de ácido gálico/g muestra), siendo ligeramente mayor para C. officinalis, lo que permitió la obtención de extractos enriquecidos en compuestos fenólicos con acción antioxidante. Los aceites esenciales de E. pellita y C. officinalis no inhibieron el crecimiento de los microorganismos evaluados. Los extractos de E. pellita y de C. officinalis a 20 y $50 \mathrm{mg} / \mathrm{mL}$ inhibiéron a $S$. aureus siendo menor la inhibición causada por C.officinalis. Los bioensayos de toxicidad mostraron que el aceite esencial de E. pellita evidenció una inhibición en la germinación encontrando un EC50 de $37.41 \mu \mathrm{L}$ para las semillas de lechuga y de $28.60 \mu \mathrm{L}$ para las de trébol. Con el aceite esencial de $C$. officinalis y con los extractos de las dos especies no hubo inhibición en la germinación de las semillas bajo las concentraciones evaluadas.

\section{CONCLUSIÓN}

Se concluye que E. pellita y C. officinalis contienen polifenoles y flavonoides con potencial antioxidante útil en el control de enfermedades crónicas y degenerativas; el contenido de lípidos, proteínas y fibra cruda refleja un aporte nutricional como suplemento en la dieta animal y la capacidad herbicida del aceite de E. pellita implica un uso potencial en el control biológico de malezas.

\section{PALABRAS CLAVE}

Eucalyptus pellita, Copaifera officinalis, bioactividad, toxicidad, principios activos. 


\title{
UNA APROXIMACIÓN AL DESARROLLO HISTÓRICO DE LA ETNOBOTÁNICA MEDICINAL PERUANA
}

\author{
Albán, Joaquina; Lopez, I; Cochachin, E; Castillo, H; Melchor, B; Hurtado, J. \\ Dpto. de Etnobotánica y Botánica Económica. Museo de Historia Natural. UNMSM. Avenida Arenales 1256. Lima 14, Perú; \\ jalbanc@yahoo.es
}

\section{INTRODUCCIÓN}

Cada día la investigación Etnobotánica sobre las plantas medicinales cobra mayor interés en el Perú. Tal hecho encuentra su explicación en diferentes motivaciones: la constatación de que el conocimiento, manejo y utilización tradicional de los recursos biológicos están sustentados en la sabiduría tradicional de las comunidades nativas y campesinas del Perú, en el eminente peligro de erosión cultural con la consecuente pérdida del conocimiento tradicional sobre el uso de las plantas, y sobre todo en aquellas comunidades sometidas a rápidos procesos de aculturación; la erosión biológica y la consecuente degradación de los bosques y pérdida de la biodiversidad en aquellos ecosistemas más explotados; y en el vacío de información sobre el impacto de la extracción de ciertas plantas medicinales de uso comercial, y en particular sobre el estado de las poblaciones en su hábitat natural.

El presente estudio tuvo como objetivo evaluar el actual estado actual del conocimiento etnobotánica- medicinal realizado en el Perú, analizando el desarrollo histórico de la Etnobotánica desde la perspectiva de la utilización de la flora en la categoría de uso medicinal y sus distintas subcategorías de dolencias, describiendo las temáticas desarrolladas, los autores, frecuencia de publicaciones, regiones del país y etnias con mayor incidencia de estudios.

\section{METODOLOGÍA}

La metodología aplicada se basó en la revisión de fuentes escritas y orales, examinándose las investigaciones efectuadas y las que están en curso por los investigadores nacionales y extranjeros; bibliografía publicada y bases de datos especializada. Se procesó alrededor de 700 trabajos publicados, 2.000 exsiccatae del Herbario USM y resultados de las investigaciones realizadas por los autores.

\section{RESULTADOS Y DISCUSIÓN}

Los resultados muestran que en el Perú, el conocimiento sobre los usos medicinales de las especies botánicas se dieron en cuatro momentos históricos, iniciados en el siglo XVI a partir de los testimonios orales registrados en los manuscritos y crónicas, seguidos por las recolecciones botánicas iniciadas en el siglo XVIII con la búsqueda del oro amargo (Cinchona spp) para el tratamiento de la malaria; continuadas en el siglo XIX con el establecimiento de la disciplina Etnobotanica como ciencia ocupando su lugar en la academia científica y seguida hasta la fecha (siglos XX-XXI) con las contribuciones de los investigadores en revistas indexadas y de difusión en el ámbito nacional e internacional. Al menos se conoce a la fecha que el $30 \%$ de las especies de la flora peruana poseen algún uso medicinal, y solo entre el 1-2\% los compuestos bioactivos que validan su eficacia. Los estudios etnográficos medicinales, con énfasis en la descripción del uso y forma de uso de las plantas, ha sido el que en gran medida ha sido desarrollado por los investigadores nacionales. La cuantificación de los datos medicinales- culturales es la tendencia actual a través del conocimiento del valor de uso e importancia relativa.

\section{CONCLUSIONES}

En la actualidad, la investigación Etnobotánica-medicinal cumple un rol muy importante en el estudio y descubrimiento de nuevos fármacos y otros recursos vegetales utilizados para satisfacer las necesidades básicas de las comunidades campesinas y nativas peruanas. La investigación etnobotánica deberá entonces, responder a las exigencias del mundo actual, aprovechando la biodiversidad que cada área geográfica nacional ofrece, en el marco de un desarrollo sustentable.

\section{FINANCIAMIENTO}

Fondo de desarrollo universitario (FEDU). UNMSM. 


\title{
ETHNOBOTANY IN THE ITALIAN ALPS: TRADITIONAL USES OF PLANTS AMONG THE INHABITANTS OF THE ALTA VALTELLINA (SONDRIO, LOMBARDY)
}

\author{
Sara Vitalini ${ }^{1}$, Franca Tomè2, Marcello Iriti $^{1}$
}

1. Dipartimento di Scienze Agrarie e Ambientali - Produzione, Territorio, Agroenergia, Università degli Studi di Milano, via Celoria 2, 20133 Milano, Italy

2. Dipartimento di Bioscienze, Università degli Studi di Milano, via Celoria 26, 20133 Milano, Italy

\section{INTRODUCTION}

The folk knowledge on the medicinal and gastronomic use of autochthonous plant species in the North Italy has been till now poorly documented, unlike what happened in Central-Southern regions [1]. Therefore, the aim of the present work was to explore the traditional plant knowledge of an area - known as Alta Valtellina (SO, Italy) characterized by a network of villages connected by the highest passes in the Central-Eastern Alps.

\section{METHODOLOGY}

All informants were residents and the interviews were carried out in families living in each municipality of the study area. The semi-structured questionnaire was submitted to people belonging to three different generations (parents, children, grandchildren), in order to document any loss of ethnobotanical information.

\section{RESULTS}

In the preliminary phase, a total of 86 botanical taxa were recorded. The most frequently utilized plant parts were the leaves $(32 \%)$, followed by flowers $(17 \%)$, fruits $(11 \%)$ and underground part (root/rhizome/tuber) (8\%). The most common preparations were infusion and decoction, both for internal and external administration - oral use $(60 \%)$, compress (18\%), bath (7.5\%) and fumigation (3\%). Data include medicinal (67\%), alimentary (52\%), veterinary $(9.5 \%)$, cosmetic $(7 \%)$, religious and ritual $(4 \%)$, domestic and other uses (7\%). Medicinal plants are employed to treat a wide range of diseases, mainly disorders of digestive system, respiratory and genital-urinary traits, skin. The gathered data show a decrease of this information from one generation to another, both in terms of quantity and quality. For example, the traditional plant knowledge of the last (third) generation was very restricted. In some case, it fell by $70-80 \%$. It is evident that a remarkable process of erosion is taking place, and the Alta Valtellina, as other alpine areas [2,3], with changes in lifestyle habits as well as environmental conditions, faces the risk and the damage of losing this treasure of knowledge in the near future.

\section{CONCLUSIONS}

In this context, the recovery of the local traditions might be pivotal to motivate people to know and safeguard their natural resources. In conclusion, in our opinion, a study like this could be the basis for a subsequent research on the species interesting for the potential use of their active metabolites. For example, further studies on Achillea moschata Jacq., the most quoted species, never investigated about its possible pharmacological activity, are required to validate experimentally its local uses.

\section{REFERENCES}

[1] Vitalini, S., Iriti, M., Puricelli, C., Ciuchi, D., Segale, A., Fico, G., 2013. Traditional knowledge on medicinal and food plants used in Val San Giacomo (Sondrio, Italy) - An alpine ethnobotanical study. Journal of Ethnopharmacolgy 145, 517-529.

[2] Vitalini, S., Tomè, F., Fico, G., 2009. Traditional uses of medicinal plants in Valvestino (Italy). Journal of Ethnopharmacology. 121, 106-116.

[3] Pieroni, A., Giusti, M.E., 2009. Alpine ethnobotany in Italy: traditional knowledge of gastronomic and medicinal plants among the Occitans of the upper Varaita valley, Piedmont. Journal of Ethnobiology and Ethnomedicine. 5, 32. 


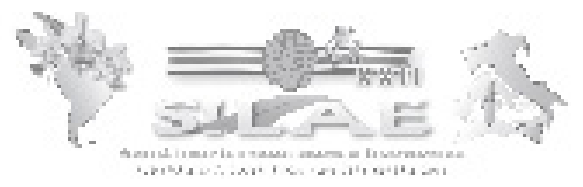

\title{
ESPECIES DE SELVA BAJA CADUCIFOLIA CON POTENCIAL EN LA ALIMENTACIÓN Y COMO FUENTES DE METABOLITOS SECUNDARIOS
}

\author{
Kalina Bermúdez Torres \\ Instituto Politécnico Nacional, Centro de Desarrollo de Productos Bióticos (CEPROBI-IPN). Calle CEPROBI No. 6, Col. San \\ Isidro, C.P. 62731 Yautepec, Morelos, México. Tel: *52557296000 Ext.52528, E-Mail: kbermudes@ipn.mx
}

\section{INTRODUCCIÓN}

Debido a sus características climáticas y edafológicas, en México la Selva Baja Caducifolia (SBC) fue elegida por las grandes culturas para establecerse y hacer florecer sus ciudades (Sánchez-Azofeifa et al., 2005). Estas culturas supieron aprovechar la gran diversidad biológica de este ecosistema, dando origen a cultivos que hoy son la base de nuestra alimentación. Estos cultivos se originaron en el territorio mexicano y fueron domesticados por nuestros antepasados. El maíz, Zea mays (Matsuoka et al. 2002), el frijol, Phaseolus vulgaris (Gepts, 1998), el chile, Capsicum annuum (Hernández-Verdugo et al., 1999), la calabaza, Cucurbita pepo (Decker-Walters et al., 1993), el girasol, Helianthus annuus (Bye et al., 2009) y el algodón, Gossypium hirsutum (Brubaker y Wendel, 1994) son producidos en el mundo y han definido la economía de muchos países. Actualmente, en nuestro país la SBC continua abasteciendo a las comunidades de medicamentos, alimentos, materiales para la construcción, plantas de ornato, combustible, forrajes, escobas, cercos vivos, jícaras, artesanías, resinas etc. (Maldonado, 1997). El objetivo del presente trabajo fue el de evaluar a partir de datos bibliográficos el potencial en la alimentación y como fuente de metabolitos secundarios de especies arbóreas de SBC.

\section{METODOLOGÍA}

Se realizó una revisión bibliográfica de artículos, libros y tesis publicados en los últimos 20 años que tuvieron como objetivo la evaluación del potencial alimentario o como fuente de metabolitos secundarios.

\section{RESULTADOS Y DISCUSIÓN}

Rzedowski (2013) reporta que las tres principales familias de la SBC son Fabaceae, Asteracea, Poaceae con 85, 78 y 41 géneros, respectivamente. Las especies de SBC encuentran diversos usos, Maldonado (1997) reporta que de las 421 especies de la SBC reportadas para la Sierra de Huautla, Morelos el 71.25\% tienen un uso medicinal, $28.97 \%$ son comestibles, $18.28 \%$ se utilizan para la construcción, $16.38 \%$ como plantas de ornato, $10.68 \%$ son usadas como combustible, $9.5 \%$ como plantas forrajeras y en menor porcentaje como cercos vivos, escobas, relleno de almohadas, bateas, jícaras, estropajos, artesanías, extracción de resinas, como curtientes, en diversos rituales, plantas tintóreas, empleadas como tutores e insecticidas. Según los diferentes autores y las zonas evaluadas la clasificación de los usos vario entre 7 y 12, siendo el uso medicinal y el alimenticio los de mayor relevancia. Se realizaron registros de los estudios sobre las especies con mayor uso en la alimentación y en la medicina/agricultura, y se correlacionaron con los contenidos de proteínas, carbohidratos, grasas, etc., así como de compuestos con actividad biológica.

\section{CONCLUSIONES}

La SBC es una fuente importante de recursos para la alimentación y la medicina/agricultura.

La SBC ha dado origen a numerosas especies de plantas que hoy en día cubren nuestros requerimiento de proteínas y carbohidratos.

Las especies silvestres arbóreas de SBC representan un importante potencial para la alimentación y la obtención de metabolitos secundarios.

\section{FINANCIAMIENTO}

Kalina Bermúdez Torres son becarios de COFAA y EDI. 


\section{BIBLIOGRAFÍA}

Brubaker C.L. \& Wendel J. F. 1994. Reevaluating the origin of domesticated cotton (Gossypium hirsutum; Malvaceae) using nuclear restriction fragment length polymorphisms (RFLPs). American journal of botany. 81(10) p. 1309-1326.

Bye, R., Linares, E., Lentz, D. L. 2009. México: Centro de Origen de la domesticación del Girasol. Revista Especializada En Ciencias Químico-Biológicas, Vol. 12, Núm. 1: 5-12

Decker-Walters, D.S., T.W. Waters, C.W., Cowan, and B.D. Smith. 1993 Isozymic characerization of wildpopulatiosn of Cucurbita pepo. J. Ethnobiol. 13:55-72

Gepts P. 1988. Genetic resources of Phaseolus beans. Eds. P. Gepts. Kluwer Academic Publishers. Dordrecht, Boston, London. 375-391.

Hernández-Verdugo, S., P. Dávila-Aranda, y K. Oyama. 1999. Síntesis del conocimiento taxonómico, origen y domesticación del género Capsicum. Bol. Soc. Bot. Méx. 64: 65-84.

Matsuoka Y., Vigouroux Y.,. Goodman M., Sanchez J., Buckler E. and Doebley J. 2002. A single domestication for maize shown by multilocus microsatellite genotyping. Proceedings of the National Academy of Sciences 99: 6080-6084.

Maldonado, 1997. Aprovechamiento de los recursos florísticos de la Sierra de Huautla, Morelos. México. Tesis de Maestría. UNAM, México.

Sánchez-Azofeifa G.A., Quesada M., Rodríguez J.P.., Nassar J. M. Stoner K.E., Castillo A, Garvin T.,Zent E.L., Calvo - Alvarado J.C., Kalacska M.E.R., Fajardo L., Gamon J.A., Cuevas - Reyes P., 2005. Research priorities for neotropical dry forests. Biotropica $37,477-485$ 


\title{
ACTIVIDAD ANTI-INFLAMATORIA Y ANTIOXIDANTE in vitro DE LOS EXTRACTOS OBTENIDOS DE Tabebuia rosea (BERTOL) DC. (BIGNONIACEAE)
}

\author{
Vélez, J.M. ${ }^{1}$; Jiménez-González, F.J.²; Veloza, L.A. ${ }^{3}$; Sepúlveda-Arias, J.C. ${ }^{4}$ \\ 1. BSc, Universidad Tecnológica de Pereira/Grupo Polifenoles, La Julita, Pereira, Colombia. \\ 2. Estudiante Doctorado en Ciencias Biomédicas, Universidad Tecnológica de Pereira/Grupo Polifenoles, La Julita, Pereira, Colombia. \\ 3. PhD, Universidad Tecnológica de Pereira/Grupo Polifenoles, La Julita, Pereira, Colombia. \\ 4. MD., PhD, Universidad Tecnológica de Pereira/Grupo Infección e Inmunidad, La Julita, Pereira, Colombia; jcsepulv@utp.edu.co
}

\section{INTRODUCCIÓN}

Tabebuia spp. (Bignoniaceae) son nativas de los bosques húmedos tropicales de Centro y Sudamérica y han sido utilizadas en medicina tradicional para el tratamiento de infecciones bacterianas y enfermedades de origen inflamatório. Tabebuia rosea, se ha empleado en medicina tradicional como agente antiinfeccioso y en el tratamiento de mordeduras de serpiente. Existe solo un reporte en la literatura indicando que los extractos y algunas fracciones obtenidas de T.rosea tienen actividad anti-inflamatoria in vivo y antioxidante in vitro. Se han aislado metabolitos secundarios de su corteza interna tales como naftoquinonas, $\alpha$-lapachona, $\alpha$-lapachona, y tabebuialdehidos A y $\mathrm{C}$ entre otros. Debido a la poca información disponible con relación a los componentes y al efecto anti-inflamatorio y antioxidante de los extractos vegetales de la especie T. rosea, se evaluó dichas actividades a los extractos obtenidos de las hojas y corteza interna de T. rosea en un modelo in vitro.

\section{METODOLOGÍA}

Tanto las hojas de T. rosea $(1.3 \mathrm{Kg})$ como su corteza interna $(2.0 \mathrm{Kg})$, secas y molidas, se extrajeron por percolación con metanol durante seis días a temperatura ambiente. Se realizaron extracciones sucesivas líquido- líquido con diferentes solventes (n-hexano, cloroformo, acetato de etilo, butanol y agua). Los extractos obtenidos se concentraron a presión reducida y se emplearon para los ensayos in vitro. Se evaluó la citotoxicidad de los extractos sobre la línea de macrófagos murinos RAW264.7 con el método del MTT. Se estimularon las células RAW264.7 con lipopolisacárido bacteriano (LPS) a concentraciones de 5 y $10 \mu \mathrm{g} / \mathrm{mL}$ durante 12 o 18 horas, con el fin de determinar la producción de $\mathrm{PGE}_{2} / \mathrm{TNF}-\alpha / \mathrm{IL}-10$ y NO, respectivamente. Para la evaluación de la actividad antioxidante, se prepararon los extractos a concentraciones de 500 y $1000 \mu \mathrm{g} / \mathrm{mL}$, empleando como solvente metanol grado analítico. Se empleó una solución de trabajo de DPPH a $20 \mu \mathrm{g} / \mathrm{mL}$. Se utilizaron como controles positivos ácido ascórbico, ácido gálico y Trolox a 5, 25, 50, 100 y $200 \mu \mathrm{g} / \mathrm{mL}$. Todas las soluciones resultantes se mezclaron con el DPPH y fueron incubadas en oscuridad durante 30 minutos a temperatura ambiente y se leyó la absorbancia a $517 \mathrm{~nm}$.

\section{RESULTADOS / DISCUSIÓN / CONCLUSIONES}

Ninguno de los extractos evaluados mostró efectos citotóxicos superiores al $80 \%$ en concentraciones menores o iguales a $2.5 \mathrm{\mu g} / \mathrm{mL}$. Los extractos en cloroformo y n-hexano obtenidos a partir de las hojas de T. rosea mostraron el mayor efecto inhibitorio sobre la producción de $\mathrm{PGE}_{2}$ y NO. De otro lado, el extracto en cloroformo obtenido a partir de la corteza interna, inhibió la producción de TNF- $\alpha$. La actividad captadora del radical DPPH fue promisoria para el extracto en acetato de etilo obtenido a partir de las hojas, con un IC50 de $157.5 \pm 2.35 \mu \mathrm{g} /$ $\mathrm{mL}$. Los resultados obtenidos indican que el extracto en cloroformo obtenido tanto a partir de las hojas como de la corteza interna de T. rosea, es promisorio dado su efecto inhibitorio sobre la producción de varios mediadores inflamatorios. En términos de actividad antioxidante, solo el extracto en acetato de etilo es promisorio.

\section{AGRADECIMIENTOS}

Se agradece a COLCIENCIAS por el financiamiento de la joven Investigadora J.M. Vélez y el soporte financiero de la Vicerrectoría de Investigaciones Innovación y Extensión de la Universidad Tecnológica de Pereira. 


\title{
COMPUTERIZED SYSTEM OF GEOREFERENCING AND ACCESS TO BIODIVERSITY WITH APPLICATION IN THE SEARCH FOR BIOACTIVE NATURAL PRODUCTS FOR MEDICINE AND AGRICULTURE
}

\author{
Gustavo L. Rosado ${ }^{1}, 2$, Hugo Rody ${ }^{1}, 2$, Charles A. A. S. Ribeiro ${ }^{1},{ }^{3}$, Marcos V.R.C Simão1 ${ }^{3}$, Andressa V. \\ Meri' ${ }^{1}$ 4, Prímula V. Campos ${ }^{1}$,4, João Paulo V. Leite ${ }^{1},{ }^{2}$ \\ 1. Universidade Federal de Viçosa (UFV) \\ 2. Laboratório de Biodiversidade, Departamento de Bioquímica e Biologia Molecular \\ 3. Departamento de Engenharia Florestal, 4Departamento de Biologia Vegetal, Viçosa, MG; jpleite@ufv.br
}

\section{INTRODUCTION}

According to current regulations of the Convention on Biological Diversity, countries with high biodiversity should propose goals and challenges for the conservation, monitoring and sustainable use of their natural resources (Neelakandan, 2006). In this context, because Brazil is a megabiodiverse country, it plays an important role in proposing and implementing technologies for assisting in this process. The MAPA program and database was created in order to optimize access to the genetic biome of the Atlantic Forest and for investigation of natural products in the search of bioactive molecules. This program is interdisciplinary, with participation of researchers from diverse fields of knowledge whose goal is to implement a computerized database of plant extracts from species of the Atlantic Forest (Extract library), associated with georeferencing resources (GIS) and databases with ethnopharmacological, chemical and biological information, so as to permit the search for bioproducts of interest in medicine and agriculture (Cantrell, 2012).

\section{METHODOLOGY}

For the development of the MAPA system the PHP language was used (Hypertext Preprocessor), an open source scripting language that allows for creation of web applications and compatibility with management of MySOL databases. Data from a global positioning system (GPS) are obtained by a Garmin GPSmap 60CSx unit, which are listed for assessment of species located in two Atlantic Forest fragments in the state of Minas Gerais, Brazil $\left(20^{\circ} 45^{\prime} 27.5^{\prime \prime}\right.$ S and $\left.42^{\circ} 51^{\prime} 38.7^{\prime \prime} \mathrm{W}\right)$.

\section{RESULTS AND DISCUSSION}

Currently MAPA consists of approximately 148 registered samples and more than 300 plant species native to the Atlantic Forest are in the identification process that can generate around 900 extracts, considering the production of extracts from different parts of the plant. Ethnopharmacological, pharmacological and phytochemical composition data of the identified species were collected to make up the databases. Also available in the MAPA were scanned images of herbarium specimens, maps for location the specimen in the forest and a search function for seeking species of interest for bioprospecting studies. This extract library coupled with the georeferencing system and database permit access to biodiversity with a bioprospecting characteristic, employing ethnopharmacological and chemotaxonomic strategies, or by traceability of scientific information on chemical-biological aspects of the species.

\section{CONCLUSION}

Therefore the MAPA program seeks to optimize access of researchers to genetic information and associated traditional knowledge of forest species from the Atlantic Forest biome when searching for bioactive natural products.

\section{SPONSORS}

\section{Capes, CNPq and FAPEMIG}

\section{BIBLIOGRAPHY}

Cantrell, C.L.; Dayan, F.E.; Duke, S.O. Natural Products As Sources for New Pesticides. Journal of Natural Products. n.75, p.1231-1242, 2012.

Neelakandan, V.N.; Mohanan, C.N.; Sukumar, B. Development of a Biogeographical Information Systems for Conservation Monitoring of Biodiversity. Current Science. v.90, n.3, p.10, 2006. 